\title{
The diagnostic value of shear wave elastography for parathyroid lesions and comparison with cervical lymph nodes
}

\author{
Ahmet Veysel Polat ${ }^{1}$, Mesut Ozturk ${ }^{1}$, Behic Akyuz ${ }^{1}$, Cetin Celenk ${ }^{1}$, Mehmet Kefeli², \\ Cafer Polat ${ }^{3}$
}

${ }^{1}$ Department of Radiology, ${ }^{2}$ Department of Pathology, ${ }^{3}$ Department of General Surgery, Faculty of Medicine, Ondokuz Mayis University, Samsun, Turkey

\begin{abstract}
Aims: The aim of this study was to prospectively assess the feasibility of the Virtual Touch tissue imaging quantification (VTIQ) method of shear wave elastography (SWE) for the discrimination of parathyroid lesions and to compare the lesions' stiffness with that of cervical lymph nodes. Materials and methods: SWE using VTIQ was performed on 66 patients with 87 histopathologically proven parathyroid lesions (54 parathyroid adenomas and 33 parathyroid hyperplasia) and 29 patients with 31 inflammatory cervical lymph nodes. The mean SWVs of the lesions were compared and receiver operating characteristic (ROC) curve analysis was used to evaluate diagnostic performance. Results: The mean SWV of parathyroid adenomas $(2.16 \pm 0.33 \mathrm{~m} / \mathrm{s})$ differed significantly from those of parathyroid hyperplasia and lymph nodes $(1.75 \pm 0.28 \mathrm{~m} / \mathrm{s}$ and $1.86 \pm 0.37$ $\mathrm{m} / \mathrm{s}$ respectively, $\mathrm{p}<0.001$ ). Selecting a cutoff value of $1.92 \mathrm{~m} / \mathrm{s}$ for diagnosing adenoma led to $80 \%$ sensitivity and $82 \%$ specificity (area under the curve [AUC]: 0.832 [95\% confidence interval (CI): 0.742-0.921], p<0.001). Conclusions: The VTIQ method of SWE can contribute to the differentiation of parathyroid adenoma from parathyroid hyperplasia and cervical lymph nodes.
\end{abstract}

Keywords: parathyroid adenoma; shear wave elastography; lymph nodes; parathyroid hyperplasia; ultrasonography

\section{Introduction}

Primary hyperparathyroidism is a clinical situation characterized by the excessive secretion of the parathyroid hormone $(\mathrm{PTH})$ leading to increased serum calcium levels. It is more common in women and is more prevalent in the fifth to seventh decade of life [1]. Most cases occur due to a single adenoma (88\%); other less common causes are multiglandular disease from double adenoma (4\%), multiple gland hyperplasia $(6 \%)$, and rarely carcinoma $(<1 \%)[1-3]$. Surgical excision of the abnormal gland or glands offers a definitive, curative treatment. Appropriate preoperative localization and exclusion of

Received 08.05.2017 Accepted 23.08.2017

Med Ultrason

2017, Vol. 19, No 4, 386-391

Corresponding author: Ahmet Veysel Polat, M.D.

Ondokuz Mayis University, Faculty of Medicine, Department of Radiology, 55139 Kurupelit,

Samsun, Turkey

Phone: +90-362-312-1919

E-mail: veyselp@hotmail.com the multiglandular disease help to determine the surgical approach.

Ultrasonography (US) is a non-invasive, cheap, and widely used imaging modality employed to evaluate preoperatively the patients with hyperparathyroidism. The sensitivity of US in determining the site of the parathyroid adenoma varies from $74 \%$ to $89 \%$ [2]. High-resolution grayscale images, use of power Doppler US, and the radiologist's experience may increase its sensitivity $[2,4]$. However, despite the significant progress in the image quality and scientific knowledge using this modality, it may still be challenging to differentiate a parathyroid lesion from other neck lesions, such as cervical lymph nodes or thyroid nodules $[5,6]$. Basic findings that should be reported to the surgeon include the localization and size of the lesion, the presence of the multiglandular disease, and the level of confidence in the US diagnosis of parathyroid adenoma [1].

Shear wave elastography (SWE) is an US-based imaging modality that evaluates tissue stiffness [7-13]. In SWE, a vertical pressure is applied to the tissue through 
a short-duration, high-powered acoustic repulsive radiation force from the probe. This acoustic force causes horizontal displacements in tissue that are called "shear waves". Acoustic radiation force impulse (ARFI) technique of SWE estimates the elasticity of a region of interest (ROI) by measuring the speed of shear waves [13]. There are two ARFI techniques, namely Virtual Touch quantification (VTQ) and Virtual Touch tissue imaging quantification (VTIQ). Both allow tissue elasticity to be evaluated quantitatively, and tissue stiffness is measured as shear wave velocity $(\mathrm{SWV})$ in meters/second $(\mathrm{m} / \mathrm{s})$. VTIQ allows the evaluation of the stiffness of smaller tissues using a smaller ROI. Additionally, during VTIQ examination, a two-dimensional (2D) elastography color map is displayed on the box, and differences in the SWV of the tissues are expressed in different colors; this allows the correct placement of the small ROI boxes on the stiffest area. Furthermore, a 2D color shear wave quality map is also obtained, allowing the assessment of image quality. In this study, our aim was to assess the feasibility of the VTIQ method of SWE for the discrimination of parathyroid lesions and to compare the lesions' stiffness with that of cervical lymph nodes.

\section{Materials and methods}

This was a prospective, single-center study that was approved by our university's local ethics committee conducted between May 2015 - September 2016. All study participants gave informed consent before examination.

\section{Parathyroid lesions}

Patients suffering from clinical symptoms of hyperparathyroidism with biochemically proven high serum PTH and calcium levels were examined at our hospital for the etiology of hyperparathyroidism and were referred to our imaging department for US examination. Among them, 73 patients whose parathyroid lesions were detected with US were included in this study. Two patients were excluded because of inaccessible pathology results, while five patients were excluded because of low VTIQ image quality due to patient obesity or deeply located lesions. Finally the study group comprised 66 patients ( 49 women and 17 men) with a mean age of $50.25 \pm 12.64$ years.

\section{Lymph nodes}

Lymph node SWV measurements were obtained from patients with cervical lymph nodes detected during US examination for other reasons. This group consisted of 29 patients ( 20 women, 9 men). Lymph nodes were located at cervical level IV or VI. The inclusion criteria for those patients were as follows: (i) no clinical symptoms of hyperparathyroidism, (ii) normal serum calcium levels, (iii) the presence of the appearance of US reactive lymph nodes, and (iv) no history of any previous neck surgery. Lymph nodes were accepted if they were oval with a preserved fatty hilum, no asymmetrical cortical thickening, and a maximal axial diameter of less than $8 \mathrm{~mm}$ [14].

\section{US and SWE Examination}

US and SWE of the parathyroid lesions and lymph nodes were performed by a radiologist (A.V.P) with 19 years of experience in US and 5 years of experience in elastography. The examinations were performed using the Siemens ACUSON S2000 US system (Siemens Medical Solution, Mountain View, CA, USA) with a 9L4 multi-D probe. All subjects were positioned supine with the neck slightly extended. During B-mode examination, the size and location of lesions were noted. For SWE examination, the transducer was vertically placed on the skin surface with an adequate amount of gel, and a VTIQ color map was obtained without applying any pressure to the probe to avoid the compression effect. The subjects were also told to hold their breath during image acquisition to minimize breathing artifacts. A 2D elastography color map was displayed on the screen, and five small ROI boxes randomly were placed within the lesion (fig 1, fig 2). Lesion stiffness was expressed as the mean SWV of the five measurements; this mean value was used for statistical analysis. Shear wave quality maps were also obtained for each VTIQ examination; on these maps, high-quality regions were displayed as green, low-quality regions were displayed as orange, and

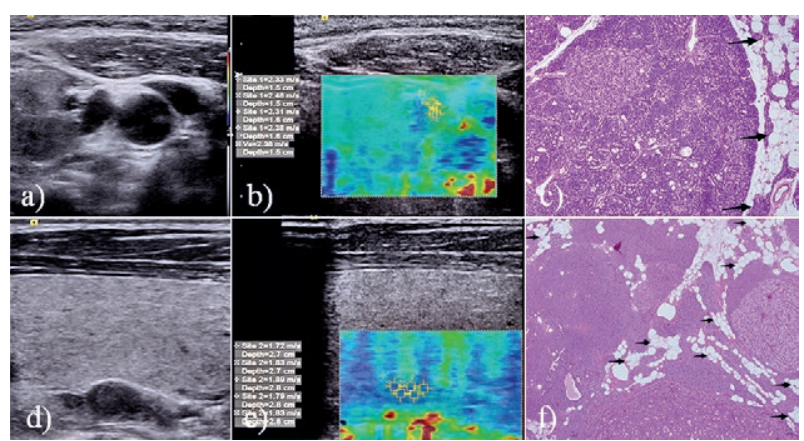

Fig 1. Ultrasound (US) and shear wave elastography (SWE) examination, and pathology of a parathyroid adenoma (a-c) and parathyroid hyperplasia (d-f) are shown: a) grayscale axial US image shows an oval hypoechoic parathyroid adenoma located in between the left lobe of thyroid gland and left carotid artery; b) shear wave velocity (SWV) measurements of the lesion; c) pathology of the lesion shows a rim of normal parathyroid (arrows: parathyroid rim; hematoxylin and eosin $\times 40$ ); d) grayscale sagittal US image shows an oval hypoechoic parathyroid hyperplasia located posterior to the right thyroid lobe; e) SWV measurements of the lesion; f) gland shows a multinodular growth pattern composed of populations of mainly chief cells and clear cells. There are small clusters of adipocytes between nodules (arrows: clusters of adipocytes; hematoxylin and eo$\sin \times 40)$. 
marginal-quality regions were displayed as yellow [15]. All ROI boxes were placed within the green areas on the shear wave quality map.

\section{Surgery and histopathological examination}

Surgical excision was performed on all parathyroid lesions and the final diagnosis was based on pathology results. Surgical excision of all lesions was performed by a surgeon (C.P.) with 20 years of experience in parathyroid and thyroid surgery. During B-mode examination of the patients, the surgeon was also present in the examination room for preoperative US localization of the lesions.

\section{Statistical analysis}

The statistical analyses were performed with the Statistical Package for the Social Sciences 18.0 (SPSS Inc.; Chicago, IL, USA) for Windows. Data are presented as means and standard deviations for continuous variables. The differences in the mean SWVs of the lesions were evaluated using the one-way analysis of covariance (ANCOVA) test. Age and lesion size were included in the model as covariate. Demographic data of the population and clinical characteristics were analyzed using Chi-square test, Kruskal-Wallis test, and Mann-Whitney $\mathrm{U}$ test, as appropriate. A p-value $<0.05$ was indicative of a statistically significant difference. ROC curves were constructed to assess the diagnostic performance of SWE in differentiating parathyroid adenoma from parathyroid hyperplasia and lymph nodes, and the best cutoff value to differentiate parathyroid adenoma was obtained. The

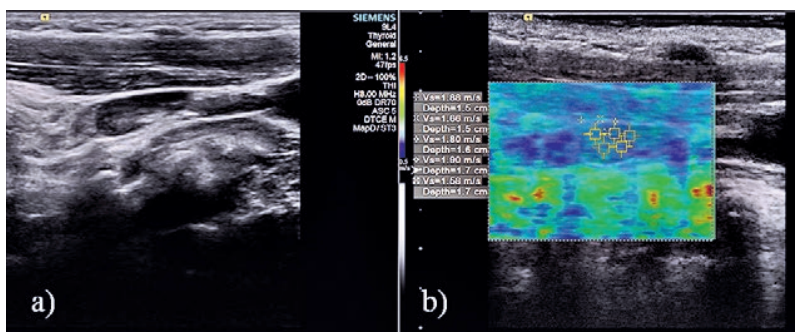

Fig 2. Ultrasound (US) and shear wave elastography (SWE) examination of a left sided level IV lymph node: a) grayscale US image shows an oval, hypoechoic lesion with a preserved fatty hilum; b) shear wave velocity (SWV) measurements of the lesion are shown. sensitivity and specificity for each comparison were calculated.

\section{Results}

Comparison of the mean SWVs of the lesions and demographic data of the included patients are displayed in Table I. A total of 87 parathyroid lesions and 31 lymph nodes were examined. According to the postsurgical results, 54 lesions were diagnosed as parathyroid adenoma and 33 were diagnosed as parathyroid hyperplasia.

The analysis of mean SWV values of the lesions demonstrated that the mean SWV of the parathyroid adenoma $(2.16 \pm 0.33 \mathrm{~m} / \mathrm{s})$ was significantly higher than that of parathyroid hyperplasia or lymph nodes $(1.75 \pm 0.28 \mathrm{~m} / \mathrm{s}$ and $1.86 \pm 0.37 \mathrm{~m} / \mathrm{s}$, respectively, $\mathrm{p}<0.001$ ) (fig 3 ). However, no statistically significant difference was found between the mean SWVs of parathyroid hyperplasia and lymph nodes $(\mathrm{p}=0.365)$.

The area under the curve (AUC) for the differentiation of parathyroid adenoma and hyperplasia was $0.832(95 \%$

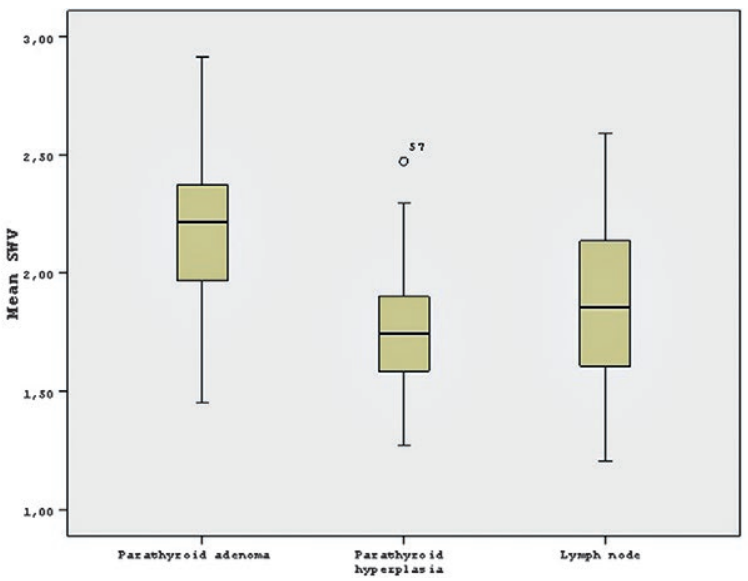

Fig 3. Box-and-whisper plot of mean shear wave velocity (SWV) of the parathyroid lesions and lymph nodes. The mean SWV of the parathyroid adenoma $(2.16 \pm 0.33 \mathrm{~m} / \mathrm{s}$; range: $1.45-$ $2.92 \mathrm{~m} / \mathrm{s}$ ) was significantly higher than that of parathyroid hyperplasia $(1.75 \pm 0.28 \mathrm{~m} / \mathrm{s}$; range: $1.27-2.47 \mathrm{~m} / \mathrm{s})$ or lymph nodes $(1.86 \pm 0.37 \mathrm{~m} / \mathrm{s}$; range: $1.21-2.59)$.

Table I. Demographic data of the study population and comparison of the mean shear wave velocity (SWV) of the lesions

\begin{tabular}{lllll}
\hline & $\begin{array}{l}\text { Parathyroid adenoma } \\
(\mathbf{n = 5 4 )}\end{array}$ & $\begin{array}{l}\text { Parathyroid hyperplasia } \\
(\mathbf{n = 3 3 )}\end{array}$ & $\begin{array}{l}\text { Lymph node } \\
(\mathbf{n = 3 1})\end{array}$ & p Value \\
\hline Number of patients (F/M) & $45 / 9$ & $4 / 8$ & $20 / 9$ & 0.002 \\
Age (mean) & $55.0 \pm 11.88$ & $42.48 \pm 9.75$ & $28.23 \pm 10.38$ & 0.884 \\
Serum PTH Level (pg/ml) & $225.55 \pm 186.45$ & $2073.4 \pm 1117.15$ & - & $<0.001$ \\
Serum Ca Level $(\mathrm{mg} / \mathrm{dl})$ & $11.30 \pm 0.75$ & $9.58 \pm 0.92$ & $9.66 \pm 0.37$ & $<0.001$ \\
Lesion size (mm) & $13.63 \pm 7.38$ & $9.95 \pm 3.03$ & $8.71 \pm 4.02$ & 0.104 \\
Mean SWV (m/s) & $2.16 \pm 0.33$ & $1.75 \pm 0.28$ & $1.86 \pm 0.37$ & $<0.001$ \\
\hline
\end{tabular}

a: Lesion size is calculated as the mean of two maximum diameters and expressed as millimeters, n: Number of lesions, PTH: Parathyroid hormone, Ca: Calcium, F/M: Female / Male 
CI: 0.742-0.921, $\mathrm{p}<0.001)$. A cutoff value of greater than $1.92 \mathrm{~m} / \mathrm{s}$ for diagnosing adenoma led to a sensitivity and specificity of $80 \%$ and $82 \%$, respectively. ROC analysis for parathyroid adenoma versus lymph nodes resulted in an AUC of 0.725 (95\% CI: 0.612-0.839, $\mathrm{p}<0.001)$. The sensitivity and specificity were found to be $80 \%$ and $58 \%$, respectively, at a threshold value of $1.92 \mathrm{~m} / \mathrm{s}$.

\section{Discussions}

In this study, we found that the VTIQ is a feasible modality in distinguishing between parathyroid adenoma, parathyroid hyperplasia, and cervical lymph nodes. Our study results demonstrated that parathyroid adenoma had a stiffer structure comparing with parathyroid hyperplasia and reactive lymph nodes.

A normal parathyroid gland is approximately $5 \times 3 \times 1$ $\mathrm{mm}$ in size and weighs $20-40 \mathrm{mg}$. The gland consists of chief cells, fibro-vascular stroma, and fat tissue [2]. Stromal fat constitutes $10-30 \%$ of the parathyroid and this proportion increases with age. Cellularity is also variable and distributed unevenly, but it decreases with age. Normal parathyroid glands can sometimes be seen with the help of high-frequency US probes, but they are often invisible due to their small size, deep positioning, and the presence of fat tissue, which makes their identification more difficult [2]. The increased size of the gland due to adenoma or hyperplasia makes the gland visible by US. In parathyroid adenomas little or no fat tissue is present while in hyperplasia the fat tissue is somewhat preserved. The characteristic hypoechoic appearance of parathyroid adenoma is caused by the hyper-cellular internal structure with a low fat content, which reduces the number of interfaces reflecting the sound [2]. In addition, parathyroid adenomas have a hard, fibrous capsule [16]. For those reasons, parathyroid adenoma is supposed to have a stiffer structure than parathyroid hyperplasia. From this hypothesis, preoperative elastographic evaluation of parathyroid lesions may be useful in distinguishing parathyroid adenoma from hyperplasia and may be effective in determining the type of surgical approach. In the presence of a single lesion with high SWV, a minimally invasive parathyroidectomy may be performed, leading to a reduced surgical morbidity, less complications and limited neck exploration. If the examined parathyroid lesion has low SWV values, bilateral surgical neck exploration due to the increased risk of multiglandular disease have to be discussed [5]. However, there is a need for further studies to demonstrate that preoperative parathyroid elastography can improve surgical outcomes.

Searching the literature, we found six studies investigating the elasticity of parathyroid lesions (Table II). In these studies, it was shown that different parathyroid pathologies may be distinguished by elastography. In a study carried out by Unluturk et al [5], the authors found that parathyroid adenoma was stiffer than parathyroid hyperplasia. The main limitation of their study was the use of strain elastography, which is an operator-dependent technique that cannot be used to perform tissue quantification [17]. Hattapoglu et al [18] reported that the mean SWV of parathyroid adenoma $(2.28 \pm 0.50 \mathrm{~m} / \mathrm{s})$ was significantly higher than that of parathyroid hyperplasia $(1.46 \pm 0.23$ $\mathrm{m} / \mathrm{s}$ ). The main limitation of their study was the presence of extremely few parathyroid hyperplasia cases and the use of the VTQ technique, which made it difficult to be used in small lesions. Isodiri et al [4] found that the mean Elastoscan Core Index (ECI) value of parathyroid adenoma was higher than that of parathyroid hyperplasia. In our study, we used the VTIQ technique, which allows smaller lesions to be evaluated and is an operator-independent technique. In addition, our number of parathyroid hyperplasia cases was higher than in previous studies.

Comparing 44 parathyroid lesions (41 adenomas and 3 hyperplasia) with thyroid nodules using VTQ, Anurad-

Table II. Previously published studies evaluating parathyroid lesions with elastography

\begin{tabular}{|c|c|c|c|c|}
\hline Study & $\begin{array}{l}\text { Parathyroid } \\
\text { adenoma (n) }\end{array}$ & $\begin{array}{l}\text { Parathyroid } \\
\text { hyperplasia (n) }\end{array}$ & $\begin{array}{l}\text { Lymph } \\
\text { node (n) }\end{array}$ & Comment \\
\hline Unluturk et al & 54 & 28 & - & $\begin{array}{l}\text { Median SR of parathyroid adenoma (3.56) was found to be } \\
\text { higher than the median SR of parathyroid hyperplasia (1.49). }\end{array}$ \\
\hline Hattapoglu et al & 32 & 4 & - & $\begin{array}{l}\text { Mean SWV of parathyroid adenoma }(2.28 \mathrm{~m} / \mathrm{s}) \text { was found to be } \\
\text { higher than the mean SWV of parathyroid hyperplasia }(1.46 \mathrm{~m} / \mathrm{s})\end{array}$ \\
\hline Isodiri et al & 15 & 29 & 14 & $\begin{array}{l}\text { Mean ECI of parathyroid adenoma (1.77) was found to be higher } \\
\text { than the mean values of parathyroid hyperplasia (1.35) and reac- } \\
\text { tive lymph nodes (1.12) }\end{array}$ \\
\hline $\begin{array}{l}\text { Chandramohan } \\
\text { et al }\end{array}$ & 41 & 3 & - & $\begin{array}{l}\text { Mean SWV of parathyroid lesions }(1.6 \mathrm{~m} / \mathrm{s}) \text { was compared with } \\
\text { benign and malignant thyroid nodules. }\end{array}$ \\
\hline Batur et al & 21 & - & - & Mean SWV of parathyroid adenoma was found to be $3.09 \mathrm{~m} / \mathrm{s}$. \\
\hline Azizi et al & 57 & - & - & Mean SWV of parathyroid adenoma was found to be $2.01 \mathrm{~m} / \mathrm{s}$. \\
\hline
\end{tabular}

n: Number of lesions. SR: strain ratio, SWV: shear wave velocity, ECI: Elastoscan Core Index 
ha et al [19] found that mean SWV values of parathyroid lesions were lower than those of benign and malignant thyroid nodules. In addition, Azizi et al [17] examined 57 parathyroid adenomas and compared with thyroid tissue. They found that parathyroid adenoma was softer than thyroid tissue; the mean SWV of parathyroid adenoma was $2.01 \pm 0.24 \mathrm{~m} / \mathrm{s}$ in this study. Batur et al [20] compared 21 parathyroid adenomas with 71 thyroid nodules and found that parathyroid adenomas were stiffer than benign thyroid nodules and more elastic than malignant thyroid nodules. In their study, the mean SWV of parathyroid adenoma was found to be $3.09 \pm 0.75 \mathrm{~m} / \mathrm{s}$. However, all lesions in these two studies were parathyroid adenomas and there were no cases of parathyroid hyperplasia.

In our experience, it is difficult to differentiate low cervical lymph nodes from parathyroid lesions. Cervical lymph nodes are usually located adjacent to the jugular vein on the lateral part of the neck, away from the thyroid tissue. Nevertheless, the lymph nodes adjacent to the carotid artery may be confused with the ectopic parathyroid gland [2]. However, the lymph nodes may sometimes be located in the midline near the lower pole of thyroid tissue, and they may resemble adenomas, especially when they have an oval hypoechoic appearance [2]. Our study results demonstrated that the higher SWV value of parathyroid adenoma may enhance our US findings to differentiate a cervical lymph node from a parathyroid adenoma. In the Isodiri et al study, the mean ECI value of lymph nodes was also found to be lower than that of parathyroid lesions, which was consistent with our results. In the studies investigating the contribution of SWE in the differentiation of malignant and benign lymph nodes, the mean SWV values of benign lymph nodes were found to be between 1.52 and $2.71 \mathrm{~m} / \mathrm{s}$ [21-25]. The mean SWV value of the lymph nodes in our study was $1.86 \mathrm{~m} / \mathrm{s}$.

Our study has some limitations. First, this was a single center study. Multicenter studies with larger populations are required to validate these findings. Secondly, the lymph nodes involved in the study were not examined histopathologically. However, there were US reactive lymph nodes detected during the US examination for other reasons in patients with normal serum calcium levels and without clinical symptoms of hyperparathyroidism. Thirdly, we did not assess interobserver and intraobserver variability of the SWV measurements. Finally, we did not compare the parathyroid lesions with the thyroid tissue or lesions.

\section{Conclusion}

Our study results confirmed that parathyroid adenoma is elastographically stiffer than parathyroid hyperpla- sia and the lymph node. SWE with the VTIQ technique may be a feasible modality for discriminating parathyroid adenoma from parathyroid hyperplasia and cervical lymph nodes.

\section{Conflict of interest: none}

\section{References}

1. Hoang JK, Sung WK, Bahl M, Phillips CD. How to perform parathyroid 4D CT: tips and traps for technique and interpretation. Radiology 2014;270:15-24.

2. Huppert BJ, Reading CC. The parathyroid glands. In:Rumack CM, Wilson SR, Charboneau JW, Levine D (eds). Diagnostic ultrasound, 4th edition, Elsevier Mosby, 2011;750-769.

3. Ruda JM, Hollenbeak CS, Stack BC Jr. A systematic review of the diagnosis and treatment of primary hyperparathyroidism from 1995 to 2003. Otolaryngol Head Neck Surg 2005; 132:359-372.

4. Isidori AM, Cantisani V, Giannetta E, et al. Multiparametric ultrasonography and ultrasound elastography in the differentiation of parathyroid lesions from ectopic thyroid lesions or lymphadenopathies. Endocrine 2017;57:335-343.

5. Unluturk U, Erdogan MF, Demir O, Culha C, Gullu S, Baskal N. The role of ultrasound elastography in preoperative localization of parathyroid lesions: a new assisting method to preoperative parathyroid ultrasonography. Clin Endocrinol (Oxf) 2012;76:492-498.

6. Barbaros U, Erbil Y, Salmashoglu A, et al. The characteristics of concomitant thyroid nodules cause false-positive ultrasonography results in primary hyperparathyroidism. Am J Otolaryngol 2009;30:239-243.

7. Bekci T, Ceyhan Bilgici M, Genc G, Tekcan D, Veysel Polat A, Tomak L. Evaluation of renal parenchyma elasticity with acoustic radiation force impulse quantification in nutcracker syndrome and comparisons with gray scale Doppler sonography and laboratory findings. J Ultrasound Med 2017;36:61-67.

8. Hamidi C, Goya C, Hattapoglu S, et al. Acoustic Radiation Force Impulse (ARFI) imaging for the distinction between benign and malignant thyroid nodules. Radiol Med 2015; 120:579-583.

9. Teke M, Goya C, Teke F, et al. Combination of virtual touch tissue imaging and virtual touch tissue quantification for differential diagnosis of breast lesions. J Ultrasound Med 2015;34:1201-1208.

10. Ianculescu V, Ciolovan LM, Dunant A, et al. Added value of Virtual Touch IQ shear wave elastography in the ultrasound assessment of breast lesions. Eur J Radiol 2014;83:773-777.

11. Xu JM, Xu XH, Xu HX, et al. Conventional US, US elasticity imaging, and acoustic radiation force impulse imaging for prediction of malignancy in thyroid nodules. Radiology 2014;272:577-586.

12. Azizi G, Keller JM, Mayo ML, et al.Thyroid Nodules and Shear Wave Elastography: A New Tool in Thyroid Cancer Detection. Ultrasound Med Biol 2015;41:2855-2865. 
13. Dudea SM, Botar-Jid C. Ultrasound elastography in thyroid disease. Med Ultrason 2015;17:74-96.

14. Bruneton JN, Balu-Maestro C, Marcy PY, Melia P, Mourou MY. Very high frequency (13 MHz) ultrasonographic examination of the normal neck: detection of normal lymph nodes and thyroid nodules. J Ultrasound Med 1994;13:87-90.

15. Tozaki M, Saito M, Benson J, Fan L, Isobe S. Shear wave velocity measurements for differential diagnosis of solid breast masses: a comparison between virtual touch quantification and virtual touch IQ. Ultrasound Med Biol 2013;39:2233-2245.

16. Herrera MF, Gamboa-Dominguez A. Parathyroid embryology, anatomy, and pathology. In: Clark O, Duh HQ (eds.), Textbook of endocrinesurgery, WB Saunders Company, Philadelphia, 1997;277-283.

17. Azizi G, Piper K, Keller JM, et al. Shear wave elastography and parathyroid adenoma: A new tool for diagnosing parathyroid adenomas. Eur J Radiol 2016;85:1586-1593.

18. Hattapoglu S, Goya C, Hamidi C, et al. Evaluation of parathyroid lesions with point shear wave elastography. J Ultrasound Med 2016;35:2179-2182.

19. Anuradha C, Abraham D, Manipadam MT, Paul TV. Can ARFI elastography be used to differentiate parathy- roid from thyroid lesions? European Congress of Radiology, March 4-8, 2015. Vienna, Austria. doi:10.1549/ ecr2015/B-0882.

20. Batur A, Atmaca M, Yavuz A, et al. Ultrasound elastography for distinction between parathyroid adenomas and thyroid nodules. J Ultrasound Med 2016;35:1277-1282.

21. Fujiwara T, Tomokuni J, Iwanaga K, Ooba S, Haji T. Acoustic radiation force impulse imaging for reactive and malignant / metastatic cervical lymph nodes. Ultrasound Med Biol 2013;39:1178-1183.

22. Meng W, Xing P, Chen Q, Wu C.Initial experience of acoustic radiation force impulse ultrasound imaging of cervical lymph nodes. Eur J Radiol 2013;82:1788-1792.

23. Azizi G, Keller JM, Mayo ML, et al. Shear wave elastography and cervical lymph nodes: Predicting malignancy. U1trasound Med Biol 2016;42:1273-1281.

24. Zhang JP, Liu HY, Ning CP, Chong J, Sun YM. Quantitative analysis of enlarged cervical lymph nodes with ultrasound elastography. Asian Pac J Cancer Prev 2014;16:7291-7294.

25. Cheng KL, Choi YJ, Shim WH, Lee JH, Baek JH. Virtual touch tissue imaging quantification shear wave elastography: prospective assessment of cervical lymph nodes. U1trasound Med Biol 2016;42:378-386. 